\title{
Signless Laplacian eigenvalue problems of Nordhaus-Gaddum type
}

\author{
Xueyi Huang ${ }^{a}$ and Huiqiu Lin ${ }^{b *}$ \\ ${ }^{a}$ School of Mathematics and Statistics, Zhengzhou University, \\ Zhengzhou, Henan 450001, P.R. China \\ ${ }^{b}$ Department of Mathematics, East China University of Science and Technology, \\ Shanghai 200237, P.R. China
}

\begin{abstract}
Let $G$ be a graph of order $n$, and let $q_{1}(G) \geq q_{2}(G) \geq \cdots \geq q_{n}(G)$ denote the signless Laplacian eigenvalues of $G$. Ashraf and Tayfeh-Rezaie [Electron. J. Combin. 21 (3) (2014) \#P3.6] showed that $q_{1}(G)+q_{1}(\bar{G}) \leq 3 n-4$, with equality holding if and only if $G$ or $\bar{G}$ is the star $K_{1, n-1}$. In this paper, we discuss the following problem: for $n \geq 6$, does $q_{2}(G)+q_{2}(\bar{G}) \leq 2 n-5$ always hold? We provide positive answers to this problem for the graphs with disconnected complements and the bipartite graphs, and determine the graphs attaining the bound. Moreover, we show that $q_{2}(G)+q_{2}(\bar{G}) \geq n-2$, and the extremal graphs are also characterized.
\end{abstract}

Keywords: signless Laplacian eigenvalue, Nordhaus-Gaddum type inequalities, interlacing, quotient matrix.

AMS Classification: 05C50

\section{Introduction}

Let $G$ be a simple graph with vertex set $V(G)=\left\{v_{1}, \ldots, v_{n}\right\}$. We denote the complement of $G$ by $\bar{G}$, the adjacency matrix of $G$ by $A(G)$, and the degree (resp. neighborhood) of a vertex $v \in V(G)$ by $d(v)$ (resp. $N_{G}(v)$ ). The Laplacian matrix and the signless Laplacian matrix of $G$ are the matrices $L(G)=D(G)-A(G)$ and $Q(G)=D(G)+A(G)$, respectively, where $D(G)=\operatorname{diag}\left(d\left(v_{1}\right), \ldots, d\left(v_{n}\right)\right)$ is the diagonal matrix of vertex degrees. The eigenvalues of $A(G), L(G)$ and $Q(G)$ are called the eigenvalues, Laplacian eigenvalues and signless Laplacian eigenvalues (Q-eigenvalues for short) of $G$, and denoted by $\lambda_{1}(G) \geq \lambda_{2}(G) \geq \cdots \geq \lambda_{n}(G)$, $\mu_{1}(G) \geq \mu_{2}(G) \geq \cdots \geq \mu_{n}(G)$ and $q_{1}(G) \geq q_{2}(G) \geq \cdots \geq q_{n}(G)$, respectively. Clearly, both $L(G)$ and $Q(G)$ are positive semidefinite matrices.

\footnotetext{
${ }^{*}$ Corresponding author.

E-mail address: huangxy@zzu.edu.cn (X. Huang), huiqiulin@126.com (H. Lin).
} 
Throughout this paper, we denote the disjoint union of graphs $G$ and $H$ by $G \cup H$, the disjoint union of $k$ 's copies of $G$ by $k G$, the join of $G$ and $H$ by $G \nabla H$ which is obtained from $G \cup H$ by connecting all edges between $G$ and $H$, and the Cartesion product of $G$ and $H$ by $G \square H$. Also, we denote the complete bipartite graph with two parts of sizes $s, t$ by $K_{s, t}$, and the path, cycle and complete graph on $n$ vertices by $P_{n}, C_{n}$ and $K_{n}$, respectively.

In [17], Nordhaus and Gaddum considered the lower and upper bounds on the sum and on the product of chromatic number of a graph and its complement. Since then, any bound on the sum or the product of an invariant in a graph $G$ and the same invariant in its complement $\bar{G}$ is called a Nordhaus-Gaddum type inequality. In 2007, Nikiforov [15] proposed the study of the Nordhaus-Gaddum type inequalities for all eigenvalues of a graph defining a function given by

$$
\max \left\{\left|\lambda_{k}(G)\right|+\left|\lambda_{k}(\bar{G})\right|:|V(G)|=n\right\}, \text { for } k=1, \ldots, n .
$$

For $k=1$, Nosal [18] showed that for every graph $G$ of order $n$,

$$
\lambda_{1}(G)+\lambda_{1}(\bar{G})<\sqrt{2}(n-1) .
$$

Nikiforov [15] presented a Nordhaus-Gaddum type result for the spectral radius of a graph:

$$
\frac{4}{3} n-2 \leq \lambda_{1}(G)+\lambda_{1}(\bar{G})<(\sqrt{2}-c) n,
$$

where $c$ is some constant not less than $10^{-7}$. After that, Csikvári [5] proved that

$$
\lambda_{1}(G)+\lambda_{1}(\bar{G}) \leq \frac{1+\sqrt{3}}{2} n \leq 1.3661 n,
$$

which improved the upper bound of Nikiforov. Moreover, Terpai [21] showed that

$$
\lambda_{1}(G)+\lambda_{1}(\bar{G})<\frac{4}{3} n-1 .
$$

For $k=2$, Nikiforov and Yuan [16] obtained that

$$
\lambda_{2}(G)+\lambda_{2}(\bar{G}) \leq-1+\frac{n}{\sqrt{2}} .
$$

Later, Brondani, de Lima and Oliveira [1] posed the following conjecture which improved the bound of Nikiforov and Yuan slightly.

Conjecture 1. Let $G$ be a graph on $n$ vertices. Then

$$
\lambda_{2}(G)+\lambda_{2}(\bar{G}) \leq-1+\sqrt{\frac{n^{2}}{2}-n+1} .
$$

Furthermore, they confirmed Conjecture 1 for some classes of graphs such as trees, $k$-cyclic graphs, regular bipartite graphs, complete multipartite graphs, generalized line graphs and exceptional graphs.

For the Laplacian eigenvalues, Zhai, Shu and Hong [23] (see also You and Liu [22]) posed the following conjecture on the Laplacian spread of graphs: 
Conjecture 2. Let $G$ be a graph of order $n \geq 2$. Then

$$
\mu_{1}(G)-\mu_{n-1}(G) \leq n-1,
$$

with equality holding if and only if $G$ or $\bar{G}$ is isomorphic to the join of an isolated vertex and a disconnected graph of order $n-1$.

Notice that the inequality in Conjecture 2 is equivalent to $\mu_{1}(G)+\mu_{1}(\bar{G}) \leq 2 n-1$ or $\mu_{n-1}(G)+\mu_{n-1}(\bar{G}) \geq 1$. Ashraf and Tayfeh-Rezaie [3] confirmed Conjecture 2 for bipartite graphs, and Chen and Das [6] confirmed Conjecture 2 for graphs with $d_{1}(G)-d_{n}(G) \leq \sqrt{n-3+2 / n}$. Very recently, Einollahzadeh and Karkhaneei [8] completely confirmed Conjecture 2 .

With regard to the signless Laplacian eigenvalues, Ashraf and Tayfeh-Rezaie [3] showed that $q_{1}(G)+q_{1}(\bar{G}) \leq 3 n-4$, which confirmed a conjecture posed by Aouchiche and Hansen [2]. In this paper, we study the similar problem on $q_{2}(G)+$ $q_{2}(\bar{G})$ as follows.

Problem 1. Let $G$ be a graph of order $n$. What are the upper bound and lower bound of $q_{2}(G)+q_{2}(\bar{G})$ ?

Note that $Q(G)+Q(\bar{G})=Q\left(K_{n}\right)$. Then by Weyl's inequality (see Lemma 2.1 below), we have $q_{2}(G)+q_{2}(\bar{G}) \geq q_{3}\left(K_{n}\right)=n-2$. It seems interesting to characterize the extremal graphs attaining this bound. So we first give the following theorem.

Theorem 1.1. Let $G$ be a graph of order $n \geq 4$. Then

$$
q_{2}(G)+q_{2}(\bar{G}) \geq n-2,
$$

with equality holding if and only if $G=K_{n}, n K_{1}, K_{1, n-1}, K_{n-1} \cup K_{1},\left(2 K_{1}\right) \nabla K_{n-2}$ or $K_{2} \cup(n-2) K_{1}$.

It is known that $q_{2}(G) \leq q_{2}\left(K_{n}\right)=n-2$. Then the first upper bound of $q_{2}(G)+q_{2}(\bar{G})$ is as follows.

Theorem 1.2. Let $G$ be a connected graph of order $n \geq 2$. Then

$$
q_{2}(G)+q_{2}(\bar{G}) \leq 2 n-4,
$$

with equality holding if and only if $G \in\left\{K_{2}, P_{4}, C_{4}\right\}$.

Interesting to us, by using the computer software SageMath v8.7 [20], we find that for $n=5$ there are only eight connected graphs (see Figure 1) with $q_{2}(G)+q_{2}(\bar{G}) \in$ $(2 n-5,2 n-4)$, but for $6 \leq n \leq 8$ there are no such graphs. Thus we pose the following problem.

Problem 2. Let $G$ be a connected graph of order $n \geq 6$. Does $q_{2}(G)+q_{2}(\bar{G}) \leq 2 n-5$ always hold? 

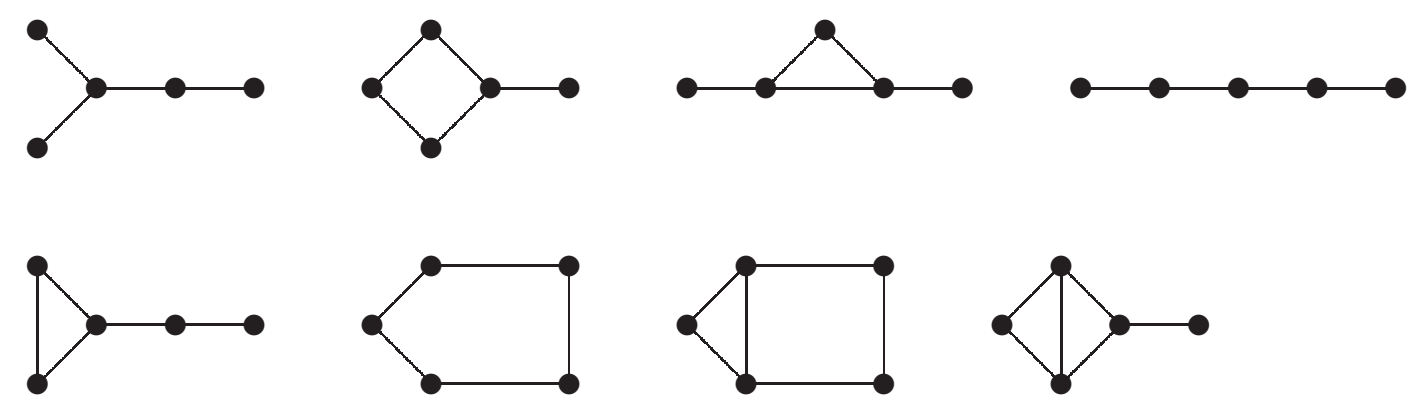

Figure 1: Connected graphs of order 5 with $q_{2}(G)+q_{2}(\bar{G}) \in(5,6)$.


Figure 2: Connected bipartite graphs satisfying $q_{2}(G)+q_{2}(\bar{G})=2 n-5$.

It is worth mentioning that for a connected regular graph $G$ of order $n \geq 6$ the inequality $q_{2}(G)+q_{2}(\bar{G}) \leq 2 n-5$ always hold. In fact, if $G=K_{n}$, then we have $q_{2}(G)+q_{2}(\bar{G})=n-2<2 n-5$. If $G$ is non-complete and $k$-regular, Chen and Das [6] proved that

$$
\mu_{1}(G)-\mu_{n-1}(G)<\sqrt{\frac{2 n k(n-k-1)}{n-1}},
$$

or equivalently,

$$
q_{2}(G)+q_{2}(\bar{G})<n-2+\sqrt{\frac{2 n k(n-k-1)}{n-1}} .
$$

From this inequality, we can deduce that $q_{2}(G)+q_{2}(\bar{G})<2 n-5$ for $n \geq 9$. For $6 \leq n \leq 8$, by using SageMath v8.7, we find that $q_{2}(G)+q_{2}(\bar{G}) \leq 2 n-5$, and $C_{6}$, $K_{3,3}, K_{3} \square K_{2}$ and $\left(2 K_{2}\right) \nabla\left(3 K_{1}\right)$ are the only connected regular graphs attaining this bound.

In order to give other positive answers to Problem 2, in this paper, we show the following three results.

Theorem 1.3. Let $G$ be a connected graph of order $n \geq 6$. If $\bar{G}$ is disconnected, then

$$
q_{2}(G)+q_{2}(\bar{G}) \leq 2 n-5,
$$


with equality holding if and only if $G$ is $\left(K_{2} \cup K_{n-3}\right) \nabla K_{1},\left(2 K_{2}\right) \nabla\left(3 K_{1}\right), K_{3,3}$ or $\left(K_{1} \cup K_{2}\right) \nabla\left(K_{1} \cup K_{2}\right)$.

Theorem 1.4. Let $G$ be a connected bipartite graph of order $n \geq 6$. Then

$$
q_{2}(G)+q_{2}(\bar{G}) \leq 2 n-5,
$$

with equality holding if and only if $G$ is one of the graphs shown in Figure 2.

Theorem 1.5. Let $G$ be a connected graph of order $n \geq 6$. If $q_{2}(G) \leq n-3$, then

$$
q_{2}(G)+q_{2}(\bar{G}) \leq 2 n-5,
$$

with equality holding if and only if $G$ is one of the graphs shown in Figure 2.

Remark 1. According to Theorems 1.3 and 1.5, to resolve Problem 1, it remains to consider the case that $G$ and $\bar{G}$ are connected graphs with $\min \left\{q_{2}(G), q_{2}(\bar{G})\right\}>n-3$.

\section{Preliminary lemmas}

Let $A$ be a Hermitian matrix of order $n$, and let $\lambda_{1}(A) \geq \lambda_{2}(A) \geq \cdots \geq \lambda_{n}(A)$ denote its eigenvalues. The following result is well known.

Lemma 2.1. (Weyl's inequality, [19].) Let $A$ and $B$ be Hermitian matrices of order $n$, and let $1 \leq i \leq n$ and $1 \leq j \leq n$. Then

$$
\begin{aligned}
& \lambda_{i}(A)+\lambda_{j}(B) \leq \lambda_{i+j-n}(A+B), \text { if } i+j \geq n+1 \\
& \lambda_{i}(A)+\lambda_{j}(B) \geq \lambda_{i+j-1}(A+B), \text { if } i+j \leq n+1 .
\end{aligned}
$$

In either of these inequalities equality holds if and only if there exists a nonzero $n$-vector that is an eigenvector to each of the three involved eigenvalues.

Let $\alpha_{1} \geq \alpha_{2} \geq \cdots \geq \alpha_{n}$ and $\beta_{1} \geq \beta_{2} \geq \cdots \geq \beta_{m}$ be two sequences of real numbers with $m<n$. The second sequence is said to be interlace the first one if

$$
\alpha_{i} \geq \beta_{i} \geq \alpha_{n-m+i} \text { for } i=1,2, \ldots, m .
$$

Lemma 2.2. (See [13].) If $B$ is a principal submatrix of a real symmetric matrix $A$, then the eigenvalues of $B$ interlace those of $A$.

Let $A$ be a real symmetric matrix of order $n$, and let $X=\{1,2, \ldots, n\}$. For any partition $\Pi: X=X_{1} \cup \cdots \cup X_{m}$, the matrix $A$ can be correspondingly partitioned as

$$
A=\left[\begin{array}{ccc}
A_{1,1} & \cdots & A_{1, m} \\
\vdots & \ddots & \vdots \\
A_{m, 1} & \cdots & A_{m, m}
\end{array}\right] .
$$

The characteristic matrix of $\Pi$ is the $n \times m$ matrix $\chi_{\Pi}$ whose columns are the characteristic vectors of $X_{1}, \ldots, X_{m}$, and the quotient matrix of $A$ with respect to $\Pi$ is the matrix $B_{\Pi}=\left(b_{i j}\right)_{m \times m}$ with $b_{i j}=\frac{1}{\left|X_{i}\right|} \mathbf{e}_{\left|X_{i}\right|}^{T} A_{i, j} \mathbf{e}_{\left|X_{j}\right|}$, where $\mathbf{e}_{\left|X_{i}\right|}$ and $\mathbf{e}_{\left|X_{j}\right|}$ are the all ones $\left|X_{i}\right|$ - and $\left|X_{j}\right|$-vectors, respectively. In particular, the partition $\Pi$ is called equitable if each block $A_{i, j}$ has constant row sum. 
Lemma 2.3. (See [4,12,13].) Let $B_{\Pi}$ be a quotient matrix of $A$ with respect to some partition $\Pi$. Then the eigenvalues of $B_{\Pi}$ interlace those of $A$. Furthermore, if the partition $\Pi$ is equitable, then all eigenvalues of $B_{\Pi}$ are also eigenvalues of $A$, and $A$ has the following two kinds of eigenvectors:

(i) the eigenvectors in the column space of $\chi_{\Pi}$, and the corresponding eigenvalues coincide with the eigenvalues of $B_{\Pi}$;

(ii) the eigenvectors orthogonal to the columns of $\chi_{\Pi}$, i.e., those eigenvectors that sum to zero on each block $X_{i}$ for $1 \leq i \leq m$.

Lemma 2.4. (See [14].) Let $G$ be a graph of order $n$, and $H$ a graph obtained from $G$ by deleting some edge. Then

$q_{1}(G) \geq q_{1}(H) \geq q_{2}(G) \geq q_{2}(H) \geq \cdots \geq q_{n-1}(G) \geq q_{n-1}(H) \geq q_{n}(G) \geq q_{n}(H) \geq 0$.

Let $G$ be a graph of order $n$, and let $S=\left\{v_{1}, \ldots, v_{s}\right\} \subseteq V(G)(s \geq 2)$ be a clique (resp. independent set) such that $N_{G}\left(v_{i}\right) \backslash S=N_{G}\left(v_{j}\right) \backslash S$ for all $i, j \in\{1,2, \ldots, s\}$. Take $\mathbf{x}_{l} \in \mathbb{R}^{n}(2 \leq l \leq s)$ as the vector defined on $V(G)$ with $\mathbf{x}_{l}\left(v_{1}\right)=1, \mathbf{x}_{l}\left(v_{l}\right)=-1$ and $\mathbf{x}_{l}(v)=0$ for $v \notin\left\{v_{1}, v_{l}\right\}$, then one can easily verify that $Q(G) \mathbf{x}_{l}=(d-1) \mathbf{x}_{l}$ (resp. $\left.Q(G) \mathbf{x}_{l}=d \mathbf{x}_{l}\right)$, where $d$ is the common degree of the vertices in $S$. Thus $d-1$ (resp. $d$ ) is a $Q$-eigenvalue of $G$ with multiplicity at least $s-1$. Then we have

Lemma 2.5. If $S(|S| \geq 2)$ is a clique (resp. independent set) of $G$ such that $N_{G}(u) \backslash S=N_{G}(v) \backslash S$ for any $u, v \in S$, then $G$ has $d-1$ (resp. d) as its $Q$ eigenvalue of multiplicity at least $|S|-1$, where $d$ is the common degree of the vertices in $S$.

Lemma 2.6. (See [10].) Let $G$ be a connected graph. Then

$$
q_{1}(G) \leq \max \left\{d(u)+\frac{1}{d(u)} \sum_{u v \in E(G)} d(v): u \in V(G)\right\},
$$

with equality holding if and only if $G$ is either semi-regular bipartite or regular.

The following lemma can be easily deduced from the Rayleigh's Principle and the Perron-Frobenius Theorem.

Lemma 2.7. Let $G$ be a connected graph of order $n$, and let $u, v$ be two vertices of $G$ which are not adjacent. Then

$$
q_{1}(G+u v)>q_{1}(G) .
$$

A connected bipartite graph is called balanced if its vertex classes have the same size, and unbalanced otherwise. Here an isolated vertex can be viewed as an unbalanced bipartite graph with an empty vertex class.

Lemma 2.8. (See [9].) If $G$ is a graph of order $n \geq 2$, then

$$
q_{2}(G) \leq n-2,
$$

with equality holding if and only if $\bar{G}$ has a balanced bipartite component or at least two bipartite components. 
Lemma 2.9. (See [7].) Let $G$ be a graph with maximum degree $d_{1}$ and second maximum degree $d_{2}$. Then

$$
q_{2}(G) \geq d_{2}-1 .
$$

If $q_{2}(G)=d_{2}-1$, then the maximum and the second maximum degree vertices are adjacent and $d_{1}=d_{2}$.

Lemma 2.10. (See [11].) If $G$ is a graph on $n(n \geq 6)$ vertices and $m$ edges, then

$$
q_{n}(G) \geq \frac{2 m}{n-2}-n+1 .
$$

\section{Proof of Theorem 1.1.}

Proof of Theorem 1.1. As $Q(G)+Q(\bar{G})=Q\left(K_{n}\right)$, by Lemma 2.1, we have

$$
q_{2}(G)+q_{2}(\bar{G}) \geq q_{3}\left(K_{n}\right)=n-2 .
$$

It remains to determine the graphs attaining the lower bound. Let $d_{1} \geq d_{2} \geq$ $\cdots \geq d_{n}$ and $\bar{d}_{1} \geq \bar{d}_{2} \geq \cdots \geq \bar{d}_{n}$ denote the degrees of $G$ and $\bar{G}$, respectively. If $d_{2}=0$ or $\bar{d}_{2}=0$, then $G$ is the empty graph $n K_{1}$ or the complete graph $K_{n}$, which obviously satisfy $q_{2}(G)+q_{2}(\bar{G})=n-2$. Now we always assume that $d_{2}, \bar{d}_{2} \geq 1$ and $q_{2}(G)+q_{2}(\bar{G})=n-2$. By Lemma 2.9 , we know that $q_{2}(G) \geq d_{2}-1$ and $q_{2}(\bar{G}) \geq \bar{d}_{2}-1$. We consider the following two cases.

Case 1. $q_{2}(G)=d_{2}-1$ and $q_{2}(\bar{G})=\bar{d}_{2}-1$;

In this situation, we have

$$
\begin{aligned}
n-2 & =q_{2}(G)+q_{2}(\bar{G}) \\
& =d_{2}-1+\bar{d}_{2}-1 \\
& =d_{2}-d_{n-1}+n-3,
\end{aligned}
$$

which gives that $d_{2}=d_{n-1}+1$. Since $q_{2}(G)=d_{2}-1$, from Lemma 2.9 we see that $d_{1}=d_{2}$, and all vertices in $G$ of degree $d_{2}$ must be adjacent to each other. Similarly, by considering the complement $\bar{G}$, we conclude that $d_{n-1}=d_{n}$, and the vertices in $G$ of degree $d_{n-1}$ cannot be adjacent to each other. Thus $G$ has only two kinds of degrees, i.e., $d_{n-1}+1$ and $d_{n-1}$, and the vertices of degree $d_{n-1}+1$ (resp. $\left.d_{n-1}\right)$ form a clique (resp. independent set). Now partition the vertex set of $G$ as $V(G)=V_{1} \cup V_{2}$, where $V_{1}=\left\{v \in V(G): d(v)=d_{n-1}+1\right\}, V_{2}=\left\{v \in V(G): d(v)=d_{n-1}\right\}$, and $\left|V_{i}\right|=n_{i}$ for $i=1,2$. Since $V_{1}$ is a clique, all vertices of $V_{1}$ have the same number, say $s$, of neighbors in $V_{2}$. Then we obtain $d_{n-1}+1=n_{1}-1+s$. Moreover, we claim that $d_{n-1} \leq n_{1}$ because $V_{2}$ is an independent set. Thus we get $s \leq 2$. If $s=0$, there are no edges between $V_{1}$ and $V_{2}$, so $d_{n-1}=0$ and $n_{1}=2$. This implies that $G$ is just the graph $K_{2} \cup(n-1) K_{1}$, which obviously satisfy $q_{2}(G)=0=d_{2}-1$, $q_{2}(\bar{G})=n-2=\bar{d}_{2}-1$ and $q_{2}(G)+q_{2}(\bar{G})=n-2$ by Lemma 2.8. If $s=1$, by counting the edges between $V_{1}$ and $V_{2}$, we have $n_{1}=d_{n-1} n_{2}$. Combining this with $d_{n-1}+1=n_{1}-1+s=n_{1}$, we obtain $d_{n-1}=1, n_{1}=n_{2}=2$, and so $G=P_{4}$. However, by simple computation, we have $q_{2}\left(P_{4}\right)+q_{2}\left(\overline{P_{4}}\right)=2 q_{2}\left(P_{4}\right)=4>2$, a 
contradiction. If $s=2$, then from $2 n_{1}=d_{n-1} n_{2}$ and $d_{n-1}+1=n_{1}-1+s=n_{1}+1$, we obtain $n_{2}=2, d_{n-1}=n_{1}=n-2$, and $G=\left(2 K_{1}\right) \nabla K_{n-2}$, which also satisfies the requirement.

Case 2. $q_{2}(G)>d_{2}-1$ or $q_{2}(\bar{G})>\bar{d}_{2}-1$.

Without loss of generality, we may assume that $q_{2}(G)>d_{2}-1$. We claim that $\bar{d}_{2}-1 \leq q_{2}(\bar{G})<\bar{d}_{2}$. In fact, if $q_{2}(\bar{G}) \geq \bar{d}_{2}$, then $d_{2}-d_{n-1}+n-2=$ $d_{2}-1+\bar{d}_{2}<q_{2}(G)+q_{2}(\bar{G})=n-2$, which gives that $d_{2}<d_{n-1}$, a contradiction. Also, from $n-2=q_{2}(G)+q_{2}(\bar{G})>d_{2}-1+\bar{d}_{2}-1=d_{2}-d_{n-1}+n-3$ we can deduce that $d_{2}=d_{n-1}$, implying that $G$ has at most three kinds of degrees. Let $V(G)=\left\{v_{1}, v_{2}, \ldots, v_{n}\right\}$ with $d\left(v_{1}\right)=d_{1}, d\left(v_{2}\right)=d\left(v_{3}\right)=\cdots=d\left(v_{n-1}\right)=d_{2}=d_{n-1}$ and $d\left(v_{n}\right)=d_{n}$. Then we can partition the vertex set as $V(G)=V_{1} \cup V_{2} \cup V_{3}$, where $V_{1}=\left\{v_{1}\right\}, V_{2}=\left\{v_{2}, v_{3}, \ldots, v_{n-1}\right\}$ and $V_{3}=\left\{v_{n}\right\}$.

Subcase 2.1. $d_{2}-1<q_{2}(G)<d_{2}$;

We claim that $v_{1}$ is adjacent to all vertices of $V_{2}$, since otherwise $\left[\begin{array}{cc}d_{1} & 0 \\ 0 & d_{2}\end{array}\right]$ will be the principle submatrix of $Q(G)$, which implies that $q_{2}(G) \geq d_{2}$ by Lemma 2.2, a contradiction. Similarly, as $q_{2}(\bar{G})<\bar{d}_{2}=n-1-d_{n-1}$, we see that $v_{n}$ is not adjacent to any vertex of $V_{2}$. Observe that $G\left[V_{1} \cup V_{2}\right] \cup K_{1}=\left(K_{1} \nabla G\left[V_{2}\right]\right) \cup K_{1}$ is a spanning subgraph of $G$, and $Q\left(K_{1} \nabla G\left[V_{2}\right]\right)$ has the quotient matrix

$$
B_{1}=\left[\begin{array}{cc}
n-2 & n-2 \\
1 & 2 d_{2}-1
\end{array}\right] \text {. }
$$

Then, by Lemmas 2.3 and 2.4, we have

$$
q_{2}(G) \geq \lambda_{2}\left(B_{1}\right)=\frac{1}{2}\left[n+2 d_{2}-3-\sqrt{n^{2}-\left(4 d_{2}-2\right) n+4 d_{2}^{2}+4 d_{2}-7}\right] .
$$

Similarly, $\overline{G\left[V_{2} \cup V_{3}\right]} \cup K_{1}=\left(\overline{G\left[V_{2}\right]} \nabla K_{1}\right) \cup K_{1}$ is a spanning subgraph of $\bar{G}$, and $Q\left(\overline{G\left[V_{2}\right]} \nabla K_{1}\right)$ has the quotient matrix

$$
B_{2}=\left[\begin{array}{cc}
n-2 & n-2 \\
1 & 2 n-2 d_{2}-3
\end{array}\right]
$$

which implies that

$$
q_{2}(\bar{G}) \geq \lambda_{2}\left(B_{2}\right)=\frac{1}{2}\left[3 n-2 d_{2}-5-\sqrt{n^{2}-\left(4 d_{2}-2\right) n+4 d_{2}^{2}+4 d_{2}-7}\right] .
$$

Combining (1), (2) and $q_{2}(G)+q_{2}(\bar{G})=n-2$, we obtain

$$
n-2 \geq 2 n-4-\sqrt{n^{2}-\left(4 d_{2}-2\right) n+4 d_{2}^{2}+4 d_{2}-7},
$$

or equivalently,

$$
4 d_{2}^{2}-(4 n-4) d_{2}+6 n-11 \geq 0 .
$$

Let $f(x)=4 x^{2}-(4 n-4) x+6 n-11$. For $n \geq 7$, we have $f(2)=f(n-3)=$ $13-2 n<0$. Thus $d_{2}<2$ or $d_{2}>n-3$ by $(3)$. Since $1 \leq d_{2} \leq n-2$, we have 
$d_{2}=1$ or $d_{2}=n-2$. If $d_{2}=1$, then $G\left[V_{2}\right]$ is the empty graph, so $G=K_{1, n-1}$ or $K_{1, n-2} \cup K_{1}$. In both case, we have $q_{2}(G)=1$, contrary to $q_{2}(G)<d_{2}=1$. If $d_{2}=n-2$, then $G=K_{1} \nabla\left(K_{n-2} \cup K_{1}\right)$ or $K_{n-1} \cup K_{1}$, which are impossible because $q_{2}\left(\overline{K_{1} \nabla\left(K_{n-2} \cup K_{1}\right)}\right)=q_{2}\left(K_{1, n-2} \cup K_{1}\right)=1=\bar{d}_{2}$ and $q_{2}\left(K_{n-1} \cup K_{1}\right)=n-3=d_{2}-1$. For $4 \leq n \leq 6$, by using SageMath v8.7, we find that there are no graphs satisfying $d_{2}-1<q_{2}(G)<d_{2}, \bar{d}_{2}-1 \leq q_{2}(\bar{G})<\bar{d}_{2}$ and $q_{2}(G)+q_{2}(\bar{G})=n-2$.

Subcase 2.2. $q_{2}(G) \geq d_{2}$.

In this situation, we claim that $q_{2}(G)=d_{2}$ and $q_{2}(\bar{G})=\bar{d}_{2}-1$, since otherwise we have $n-2=q_{2}(G)+q_{2}(\bar{G})>d_{2}+\bar{d}_{2}-1=d_{2}-d_{n-1}+n-2$, a contradiction. As in Subcase 2.1, we see that $v_{n}$ is not adjacent to any vertex of $V_{2}$ in $G$. Note that $\overline{G\left[V_{2} \cup V_{3}\right]} \cup K_{1}=\left(\overline{G\left[V_{2}\right]} \nabla K_{1}\right) \cup K_{1}$ is a spanning subgraph of $\bar{G}$, and $Q\left(\overline{G\left[V_{2}\right]} \nabla K_{1}\right)$ has the quotient matrix

$$
B_{3}=\left[\begin{array}{cc}
n-2 & n-2 \\
1 & 2 n-2 d_{2}-5+\frac{2 s}{n-2}
\end{array}\right],
$$

where $s=d_{1}$ or $d_{1}-1$ is the number of edges between $V_{1}$ and $V_{2}$ in $G$. By Lemmas 2.3 and 2.4 , we have

$$
q_{2}(\bar{G}) \geq \lambda_{2}\left(B_{3}\right)=\frac{(3 n-7)(n-2)-(2 n-4) d_{2}+2 s-\sqrt{\Delta}}{2 n-4},
$$

where $\Delta=n^{4}-\left(4 d_{2}+6\right) n^{3}+\left(4 d_{2}^{2}+28 d_{2}+4 s+13\right) n^{2}-\left(64 d_{2}+16 d_{2}^{2}+(8 s+64) d_{2}+\right.$ $20 s+12) n+16 d_{2}^{2}+(16 s+48) d_{2}+4 s^{2}+24 s+4$. Combining (4) with the fact that $q_{2}(\bar{G})=\bar{d}_{2}-1=n-2-d_{n-1}=n-2-d_{2}$, we obtain

$$
\sqrt{\Delta} \geq n^{2}-5 n+2 s+6,
$$

or equivalently,

$$
(n-2) d_{2}^{2}-\left(n^{2}-5 n+2 s+6\right) d_{2}+n^{2}-4 n+4 \geq 0 .
$$

Since $s \geq d_{1}-1 \geq d_{2}-1$, we have

$$
(n-2) d_{2}^{2}-\left(n^{2}-5 n+2\left(d_{2}-1\right)+6\right) d_{2}+n^{2}-4 n+4 \geq 0,
$$

that is,

$$
(n-4) d_{2}^{2}-\left(n^{2}-5 n+4\right) d_{2}+n^{2}-4 n+4 \geq 0 .
$$

Let $g(x)=(n-4) x^{2}-\left(n^{2}-5 n+4\right) x+n^{2}-4 n+4$. For $n \geq 8$, we have $g(2)=$ $g(n-3)=-(n-5)^{2}+5<0$, which implies that $d_{2}<2$ or $d_{2}>n-3$. Thus $d_{2}=1$ or $n-2$ due to $1 \leq d_{2} \leq n-2$. If $d_{2}=1$, then $G=K_{1, s+1} \cup\left(\frac{n-s-2}{2} K_{2}\right)$ with $0 \leq s \leq n-2$ or $G=K_{1, s} \cup\left(\frac{n-s-2}{2} K_{2}\right) \cup K_{1}$ with $1 \leq s \leq n-2$. If $s<n-2$, then $G$ has at least one balanced bipartite component $K_{2}$, implying that $q_{2}(\bar{G})=n-2$ by Lemma 2.8 , contrary to the assumption that $q_{2}(\bar{G})=\bar{d}_{2}-1=n-3$. Then $s=n-2$, and so $G=K_{1, n-1}$ or $K_{1, n-2} \cup K_{1}$. We claim that the later case cannot occurs, since otherwise $G$ has two bipartite components, which gives that $q_{2}(\bar{G})=n-3$ again by Lemma 2.8 , a contradiction. Thus we have $G=K_{1, n-1}$, 
which clearly satisfy the conditions $q_{2}(G)=d_{2}=1$ and $q_{2}(\bar{G})=\bar{d}_{2}-1=n-$ 3. If $d_{2}=n-2$, then $G\left[V_{2}\right]$ is a clique and $v_{1}$ is adjacent to all vertices of $V_{2}$, implying that $G=K_{1} \nabla\left(K_{n-2} \cup K_{1}\right)$ or $K_{n-1} \cup K_{1}$. In both cases, we can deduce a contradiction because $q_{2}\left(\overline{K_{1} \nabla\left(K_{n-2} \cup K_{1}\right)}\right)=q_{2}\left(K_{1} \cup K_{1, n-2}\right)=1>0=\bar{d}_{2}-1$ and $q_{2}\left(K_{n-1} \cup K_{1}\right)=n-3<n-2=d_{2}$. For $4 \leq n \leq 8$, by using SageMath v8.7, we find that $K_{1, n-1}$ is the only graph satisfying $q_{2}(\bar{G})=d_{2}, q_{2}(\bar{G})=\bar{d}_{2}-1$ and $q_{2}(G)+q_{2}(\bar{G})=n-2$.

Therefore, we conclude that $G=K_{1, n-1}$ under the assumption that $q_{2}(G)>$ $d_{2}-1$. Also, by considering $\bar{G}$ with $q_{2}(\bar{G})>\bar{d}_{2}-1$, we obtain $G=K_{n-1} \cup K_{1}$.

Concluding the above results, we have $q_{2}(G)+q_{2}(\bar{G})=n-2$ if and only if $G$ is $K_{n}, n K_{1}, K_{1, n-1}, K_{n-1} \cup K_{1},\left(2 K_{1}\right) \nabla K_{n-2}$ or $K_{2} \cup(n-2) K_{1}$.

We complete the proof.

\section{Proof of Theorems 1.2-1.5.}

Proof of Theorem 1.2. Notice that $Q(G)+Q(\bar{G})=Q\left(K_{n}\right)$. By Lemma 2.1, we have

$$
\left\{\begin{array}{l}
q_{2}(G)+q_{n}(\bar{G}) \leq q_{2}\left(K_{n}\right) \\
q_{2}(\bar{G})+q_{n}(G) \leq q_{2}\left(K_{n}\right)
\end{array},\right.
$$

which implies that

$$
\left\{\begin{array}{l}
q_{2}(G) \leq n-2-q_{n}(\bar{G}) \leq n-2 \\
q_{2}(\bar{G}) \leq n-2-q_{n}(G) \leq n-2
\end{array}\right.
$$

because $q_{2}\left(K_{n}\right)=n-2$ and $q_{n}(G), q_{n}(\bar{G}) \geq 0$. Summing up the two inequalities in (6) side by side, we obtain

$$
q_{2}(G)+q_{2}(\bar{G}) \leq 2 n-4,
$$

where the equality holds if and only if $q_{2}(G)=q_{2}(\bar{G})=n-2$ (and thus $q_{n}(G)=$ $q_{2}(\bar{G})=0$ ). By Lemma 2.8, this is the case that both $G$ and $\bar{G}$ contain a balanced bipartite component or at least two bipartite components. Then $G$ must be a connected balanced bipartite graph of order $n=2 s$ for some positive integer $s$. We claim that $s \leq 2$, since otherwise $\bar{G}$ contains $2 K_{s}$ as its spanning subgraph, implying that $q_{n}(\bar{G}) \geq q_{n}\left(2 K_{s}\right)=s-2 \geq 1$, contrary to $q_{n}(\bar{G})=0$. Thus $G=K_{2}, P_{4}$ or $C_{4}$. Conversely, one can check that $K_{2}, P_{4}$ and $C_{4}$ satisfy the relation $q_{2}(G)+q_{2}(\bar{G})=2 n-4$.

We compete the proof.

Proof of Theorem 1.3. By the assumption, we suppose that $\bar{G}=H_{1} \cup H_{2} \cup \cdots \cup H_{k}$, where $H_{i}$ is connected and $n_{i}=\left|V\left(H_{i}\right)\right|$ for $1 \leq i \leq k$. Assume that $n_{1} \geq n_{2} \geq$ $\cdots \geq n_{k}$. We consider the following two situations.

Case 1. $k \geq 3$; 
If $n_{1}=1$, then $\bar{G}$ is the empty graph, so $G=K_{n}$ and $q_{2}(G)+q_{2}(\bar{G})=n-2<$ $2 n-5$. Now suppose $n_{1} \geq 2$. Since $n_{1} \leq n-(k-1) \leq n-2$ and $n_{2} \leq \frac{n-1}{2}$, we have

$$
\begin{aligned}
q_{2}(\bar{G}) & \leq \max \left\{q_{2}\left(H_{1}\right), q_{1}\left(H_{2}\right), q_{1}\left(H_{3}\right), \ldots, q_{1}\left(H_{k}\right)\right\} \\
& \leq \max \left\{n_{1}-2, q_{1}\left(K_{n_{2}}\right)=2 n_{2}-2\right\} \\
& \leq \max \{n-4, n-3\} \\
& =n-3
\end{aligned}
$$

where the second inequality follows from Lemma 2.4. Moreover, we have $q_{2}(G) \leq$ $n-2$ by (6), and thus

$$
q_{2}(G)+q_{2}(\bar{G}) \leq 2 n-5 .
$$

Here the equality holds if and only if $q_{2}(\bar{G})=n-3$ and $q_{2}(G)=n-2$. If $q_{2}(\bar{G})=$ $n-3$, from (7) we know that $n_{2}=\frac{n-1}{2}\left(n\right.$ is odd). Since $n_{1} \geq n_{2}$ and $n_{3} \geq 1$, we have $k=3, n_{1}=n_{2}=\frac{n-1}{2}$ and $n_{3}=1$. Also, we see that $q_{2}(\bar{G})=q_{1}\left(H_{2}\right)=q_{1}\left(K_{n_{2}}\right)$, and so $H_{2}=K_{n_{2}}=K_{\frac{n-1}{2}}$ by Lemma 2.7. Moreover, we have $n-3=q_{2}(\bar{G}) \leq q_{1}(\bar{G})=$ $q_{1}\left(H_{1}\right) \leq q_{1}\left(K_{\frac{n-1}{2}}\right)^{2}=n-3$, which implies that $H_{1}=K_{\frac{n-1}{2}}$ again by Lemma 2.7 . Therefore, we have $\bar{G}=\left(2 K_{\frac{n-1}{2}}\right) \cup K_{1}$. By Lemma 2.8 , this gives that $q_{2}(G)<n-2$ because $n \geq 6$ is odd. Hence, we conclude that $q_{2}(G)+q_{2}(\bar{G})<2 n-5$ in this situation.

Case 2. $k=2$.

In this situation, we have $3 \leq n_{1} \leq n-1$ and $n_{2} \leq \frac{n}{2}$. We distinguish the following two subcases to discuss.

Subcase 2.1. $n_{2} \leq \frac{n-1}{2}$

We have

$$
\begin{aligned}
q_{2}(\bar{G}) & \leq \max \left\{q_{2}\left(H_{1}\right), q_{1}\left(H_{2}\right)\right\} \\
& \leq \max \left\{n_{1}-2, q_{1}\left(K_{n_{2}}\right)=2 n_{2}-2\right\} \\
& \leq \max \{n-3, n-3\} \\
& =n-3 .
\end{aligned}
$$

As above, we have $q_{2}(G)+q_{2}(\bar{G}) \leq 2 n-5$, where the equality holds if and only if $q_{2}(\bar{G})=n-3$ and $q_{2}(G)=n-2$.

Now suppose that $q_{2}(\bar{G})=n-3$ and $q_{2}(G)=n-2$. First assume that $q_{2}\left(H_{1}\right) \geq$ $q_{1}\left(H_{2}\right)$. According to (8), we have $n-3=q_{2}(\bar{G})=q_{2}\left(H_{1}\right) \leq n_{1}-2 \leq n-3$, which implies that $n_{1}=n-1, q_{2}\left(H_{1}\right)=n-3$ and $H_{2}=K_{1}$. As $q_{2}(G)=n-2$, by Lemma 2.8, the graph $H_{1}$ must be bipartite. Also, since $H_{1}$ has $n-1$ vertices and $q_{2}\left(H_{1}\right)=n-3$, we see that $\overline{H_{1}}$ has a balanced bipartite component or at least two bipartite components. If $H_{1}$ is a complete bipartite graph, say $H_{1}=$ $K_{s, n-1-s}(1 \leq s \leq n-2$ and $n \geq 6)$, then $\overline{H_{1}}=K_{s} \cup K_{n-1-s}$ cannot have two bipartite components, and so must have a balanced bipartite component, that is, $\overline{H_{1}}=K_{2} \cup K_{n-3}$. Thus we have $\bar{G}=K_{2, n-3} \cup K_{1}$, i.e., $G=\left(K_{2} \cup K_{n-3}\right) \nabla K_{1}$, which obviously satisfy the conditions $q_{2}(\bar{G})=n-3$ and $q_{2}(G)=n-2$. If $H_{1}$ is not complete bipartite, then $\overline{H_{1}}$ is connected, and so must be a balanced bipartite graph by the above arguments, which is impossible due to $n-1 \geq 5$. Now assume that 
$q_{2}\left(H_{1}\right)<q_{1}\left(H_{2}\right)$. Then we have $n-3=q_{2}(\bar{G})=q_{1}\left(H_{2}\right) \leq q_{1}\left(K_{n_{2}}\right)=2 n_{2}-2 \leq n-3$, implying that $n_{2}=\frac{n-1}{2}$ ( $n$ must be odd), $n_{1}=\frac{n+1}{2}$, and $H_{2}=K_{\frac{n-1}{2}}$ by Lemma 2.7. Also, since $q_{2}(G)=n-2$, we claim that $H_{1}$ is a balanced bipartite graph by Lemma 2.8. Furthermore, we have $q_{1}(\bar{G})=q_{1}\left(H_{1}\right)$ because $q_{2}(\bar{G})=q_{1}\left(H_{2}\right)$. Then $n-3=q_{2}(\bar{G}) \leq q_{1}(\bar{G})=q_{1}\left(H_{1}\right) \leq q_{1}\left(K_{\frac{n_{1}}{2}, \frac{n_{1}}{2}}\right)=q_{1}\left(K_{\frac{n+1}{4}, \frac{n+1}{4}}\right)=\frac{n+1}{2}$, implying that $n=7$ because $n \geq 6$ is odd. For $n=7$, since both sides of the above inequality equal to 4 , we must have $H_{1}=K_{2,2}=C_{4}$ by Lemma 2.7. Thus $\bar{G}=K_{2,2} \cup K_{3}$, i.e., $G=\left(2 K_{2}\right) \nabla\left(3 K_{1}\right)$, which obviously satisfy the relation $q_{2}(\bar{G})=4$ and $q_{2}(G)=5$.

Therefore, in this subcase, we conclude that $q_{2}(G)+q_{2}(\bar{G}) \leq 2 n-5$, where the equality holds if and only if $G=\left(K_{2} \cup K_{n-3}\right) \nabla K_{1}$ or $\left(2 K_{2}\right) \nabla\left(3 K_{1}\right)$.

Subcase 2.2. $\frac{n-1}{2}<n_{2} \leq \frac{n}{2}$.

We see that $n$ must be even and $n_{2}=\frac{n}{2}=n_{1}$. If $q_{2}(\bar{G})=q_{2}\left(H_{1}\right)$ or $q_{2}\left(H_{2}\right)$, then $q_{2}(\bar{G}) \leq \frac{n}{2}-2<n-3$, and so $q_{2}(G)+q_{2}(\bar{G})<2 n-5$. Thus we may assume that $q_{2}(\bar{G})=\min \left\{q_{1}\left(H_{1}\right), q_{1}\left(H_{2}\right)\right\}$. If $q_{2}(\bar{G})<n-3$, there is nothing to prove. We only need to consider the situation that $q_{2}(\bar{G}) \geq n-3$, from which we obtain $q_{1}\left(H_{i}\right) \geq n-3$ for $i=1,2$. Let $d_{1}$ denote the maximum degree of $H_{i}$. By Lemma 2.6, we have $q_{1}\left(H_{i}\right) \leq 2 d_{1}$, which gives that $d_{1} \geq \frac{n-3}{2}$. Thus $d_{1}=\frac{n}{2}-1$ due to $d_{1} \leq \frac{n}{2}-1$ and $n$ is even. For $i=1,2$, let $w_{i} \in V\left(H_{i}\right)$ be such that

$$
d\left(w_{i}\right)+\frac{1}{d\left(w_{i}\right)} \sum_{w_{i} v \in E\left(H_{i}\right)} d(v)=\max \left\{d(u)+\frac{1}{d(u)} \sum_{u v \in E\left(H_{i}\right)} d(v): u \in V\left(H_{i}\right)\right\} .
$$

We claim that $d\left(w_{i}\right)=d_{1}$ or $d_{1}-1$, since otherwise we get $n-3 \leq q_{1}\left(H_{i}\right) \leq$ $d\left(w_{i}\right)+\frac{1}{d\left(w_{i}\right)} \sum_{w_{i} v \in E\left(H_{i}\right)} d(v) \leq d_{1}-2+d_{1}=n-4$ by Lemma 2.6, a contradiction.

If $d\left(w_{i}\right)=d_{1}=\frac{n}{2}-1$, we obtain $\sum_{w_{i} v \in E\left(H_{i}\right)} d(v) \geq\left(\frac{n}{2}-2\right)\left(\frac{n}{2}-1\right)$ by using the fact that $n-3 \leq q_{1}\left(H_{i}\right) \leq d\left(w_{i}\right)+\frac{1}{d\left(w_{i}\right)} \sum_{w_{i} v \in E\left(H_{i}\right)} d(v)$. Thus $2 m\left(H_{i}\right) \geq$ $d\left(w_{i}\right)+\sum_{w_{i} v \in E\left(H_{i}\right)} d(v) \geq\left(\frac{n}{2}-1\right)^{2}$. If $d\left(w_{i}\right)=d_{1}=\frac{n}{2}-2$, as above, we have $\sum_{w_{i} v \in E\left(H_{i}\right)} d(v) \geq\left(\frac{n}{2}-2\right)\left(\frac{n}{2}-1\right)$. Then, for each $v \in N_{H_{i}}\left(w_{i}\right)$, we have $d(v)=\frac{n}{2}-1$. Let $w_{i}^{\prime}$ be the unique vertex that is not adjacent to $w_{i}$ in $H_{i}$. We see that $d\left(w_{i}^{\prime}\right)=\frac{n}{2}-2$ because all vertices of $N_{H_{i}}\left(w_{i}\right)$ are adjacent to $w_{i}^{\prime}$. Thus we have $H_{i}=K_{\frac{n}{2}-2} \nabla\left(2 K_{1}\right)$, and $2 m\left(H_{i}\right)=d\left(w_{i}^{\prime}\right)+d\left(w_{i}\right)+\sum_{w_{i} v \in E\left(H_{i}\right)} d(v)=\left(\frac{n}{2}-2\right)\left(\frac{n}{2}+1\right)$. For $n \geq 12$, in both cases we can deduce that

$$
q_{\frac{n}{2}}\left(H_{i}\right) \geq \frac{2 m\left(H_{i}\right)}{\frac{n}{2}-2}-\frac{n}{2}+1>1
$$

by Lemma 2.10. Thus $q_{n}(\bar{G})=\min \left\{q_{\frac{n}{2}}\left(H_{1}\right), q_{\frac{n}{2}}\left(H_{2}\right)\right\}>1$, and we have $q_{2}(G)<n-3$ according to (6). Hence, we conclude that $q_{2}(G)+q_{2}(\bar{G})<2 n-5$ because $q_{2}(\bar{G}) \leq$ $n-2$. For each even $n$ with $6 \leq n \leq 11$, by considering the condition $q_{1}\left(H_{i}\right) \geq n-3$ and using SageMath v8.7, we obtain $H_{i}=K_{\frac{n}{2}}$ or $K_{\frac{n}{2}-2} \nabla\left(2 K_{1}\right)$ for $i=1,2$. Recall that $\bar{G}=H_{1} \cup H_{2}$. By simple computation, we find that $q_{2}(G)+q_{2}(\bar{G}) \leq 2 n-5$, and the equality holds if and only if $\bar{G}=2 K_{3}$ or $2\left(K_{1} \nabla\left(2 K_{1}\right)\right)=2 K_{1,2}$, i.e., $G=K_{3,3}$ or $\left(K_{2} \cup K_{1}\right) \nabla\left(K_{2} \cup K_{1}\right)$.

Therefore, in this subcase, we conclude that $q_{2}(G)+q_{2}(\bar{G}) \leq 2 n-5$, with equality holding if and only if $G=K_{3,3}$ or $\left(K_{2} \cup K_{1}\right) \nabla\left(K_{2} \cup K_{1}\right)$.

We complete the proof. 


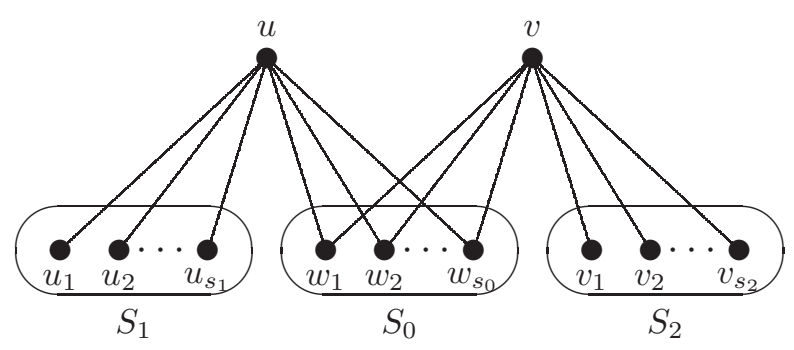

Figure 3: The graph $H\left(s_{0}, s_{1}, s_{2}\right)$.

Proof of Theorem 1.4. If $\bar{G}$ is disconnected, by Theorem 1.3 , we have $q_{2}(G)+$ $q_{2}(\bar{G}) \leq 2 n-5$, where the equality holds if and only if $G=K_{3,3}$. Now suppose that $\bar{G}$ is connected. Let $V(G)=V_{1} \cup V_{2}$ be the bipartition of $G$ with $\left|V_{i}\right|=n_{i}$ for $n=1,2$ and $n_{1} \geq n_{2}$.

If $n_{1}, n_{2} \geq 3$, then $q_{n}(\bar{G}) \geq q_{n}\left(K_{n_{1}} \cup K_{n_{2}}\right)=n_{2}-2 \geq 1$, and so $q_{2}(G) \leq n-3$ by (6). Thus we have $q_{2}(G)+q_{2}(\bar{G}) \leq 2 n-5$, where the the equality holds if and only if $q_{2}(G)=n-3$ and $q_{2}(\bar{G})=n-2$. Now assume that $q_{2}(G)=n-3$ and $q_{2}(\bar{G})=n-2$. By Lemma 2.8, the bipartite graph $G$ must be balanced, i.e., $n_{1}=n_{2}=\frac{n}{2}$. Furthermore, since $n-3=q_{2}(G) \leq q_{2}\left(K_{\frac{n}{2}, \frac{n}{2}}\right)=\frac{n}{2}$ and $n \geq 6$, we have $n=6$ and $q_{2}(G)=3$. By using SageMath v8.7, we find that all connected balanced bipartite graphs of order 6 with connected complements satisfying $q_{2}(G)=3$ and $q_{2}(\bar{G})=4$ are exactly the graphs $H_{1}-H_{7}$ shown in Figure 2 .

Next suppose $n_{2} \leq 2$. If $n_{2}=1$, then $G=K_{1, n-1}$, contrary to the connectedness of $\bar{G}$. Thus $n_{2}=2$ and $n_{1}=n-2$. Suppose $V_{2}=\{u, v\}$ and $V_{1}=S_{0} \cup S_{1} \cup S_{2}$, where $S_{0}=N_{G}(u) \cap N_{G}(v), S_{1}=N_{G}(u) \backslash N_{G}(v), S_{2}=N_{G}(v) \backslash N_{G}(u)$ and $\left|S_{i}\right|=s_{i}$ for $i=0,1,2$. Then we see that $G$ is of the form $H\left(s_{0}, s_{1}, s_{2}\right)\left(s_{0}+s_{1}+s_{2}=n-2\right)$ shown in Figure 3. By the connectedness of $G$ and $\bar{G}$, we see that $s_{0} \geq 1$ and $\max \left\{s_{1}, s_{2}\right\} \geq 1$. By the symmetry, we only need to consider the following two situation.

Case 1. $s_{1}, s_{2} \geq 1$

For $6 \leq n \leq 8$, by using SageMath v8.7, we find that $q_{2}(G)+q_{2}(\bar{G})<2 n-5$ holds for all such $G$ 's. Now suppose $n \geq 9$. If $s_{1}, s_{2} \geq 2$, then $\bar{G}$ has $2 K_{3} \cup K_{n-6}$ as its spanning subgraph, and so $q_{n}(\bar{G}) \geq q_{n}\left(2 K_{3} \cup K_{n-6}\right) \geq 1$ by Lemma 2.4 . This implies that $q_{2}(G) \leq n-3$ according to (6). Furthermore, as the connected bipartite graph $G$ is not balanced, we have $q_{2}(\bar{G})<n-2$ by Lemma 2.8, and therefore, $q_{2}(G)+q_{2}(\bar{G})<2 n-5$. It remains to consider the case that $s_{1}=1$ or $s_{2}=1$. Without loss of generality, we may assume that $s_{1}=1$. If $s_{2} \geq 2$, then $G$ is a spanning subgraph of $H(n-5,1,2)$. By Lemma 2.5, it is easy to see that the graph $H(n-5,1,2)$ has $Q$-eigenvalues 2 of multiplicity at least $n-6$ and 1 of multiplicity at least one. Furthermore, by Lemma 2.3 and the proof of Lemma 2.5, we claim that the remaining five $Q$-eigenvalues, denoted by $\alpha_{1}>\alpha_{2} \geq \alpha_{3} \geq \alpha_{4}>\alpha_{5}=0$, 
must be in the quotient matrix

$$
B_{1}=\left[\begin{array}{ccccc}
2 & 0 & 0 & 1 & 1 \\
0 & 1 & 0 & 1 & 0 \\
0 & 0 & 1 & 0 & 1 \\
n-5 & 1 & 0 & n-4 & 0 \\
n-5 & 0 & 2 & 0 & n-3
\end{array}\right] \begin{gathered}
S_{0} \\
S_{1} \\
S_{2} \\
u \\
v
\end{gathered}
$$

By simple computation, the characteristic polynomial of $B_{1}$ is $\phi\left(B_{1}, x\right)=x f(x)$, where $f(x)=x^{4}-(2 n-3) x^{3}+\left(n^{2}-n-4\right) x^{2}-\left(2 n^{2}-8 n+2\right) x+n^{2}-5 n$. Since $f(n-3)=-(n-5)(n-6)<0$, we have $\alpha_{2}<n-3$ or $\alpha_{3}>n-3$. We claim that the later case cannot occurs, since otherwise we obtain $2 n-3=\operatorname{trace}\left(B_{1}\right)=$ $\alpha_{1}+\alpha_{2}+\alpha_{3}+\alpha_{4}>3(n-3)$, which is impossible due to $n \geq 9$. Thus $\alpha_{2}<n-3$, and we have $q_{2}(G) \leq q_{2}(H(n-5,1,2))=\max \left\{\alpha_{2}, 2\right\}<n-3$. Therefore, we conclude that $q_{2}(G)+q_{2}(\bar{G})<2 n-5$. If $s_{2}=1$, then $G=H(n-4,1,1)$. As above, we see that $G$ has $Q$-eigenvalue 2 of multiplicity at least $n-5$, and the remaining five $Q$ eigenvalues, denoted by $\beta_{1}>\beta_{2} \geq \beta_{3} \geq \beta_{4}>\beta_{5}=0$, are contained in the quotient matrix

$$
B_{2}=\left[\begin{array}{ccccc}
2 & 0 & 0 & 1 & 1 \\
0 & 1 & 0 & 1 & 0 \\
0 & 0 & 1 & 0 & 1 \\
n-4 & 1 & 0 & n-3 & 0 \\
n-4 & 0 & 1 & 0 & n-3
\end{array}\right] \begin{gathered}
S_{0} \\
S_{1} \\
S_{2} \\
u \\
v
\end{gathered}
$$

By simple computation, we get $\beta_{2}=\frac{n-2+\sqrt{n^{2}-8 n+20}}{2}$, and so $q_{2}(G)=\frac{n-2+\sqrt{n^{2}-8 n+20}}{2}$. Now consider the complement graph $\bar{G}=\overline{H(n-4,1,1)}$. We see that $\bar{G}$ has $Q$ eigenvalue $n-4$ of multiplicity at least $n-5$, and the remaining five $Q$-eigenvalues lie in the quotient matrix

$$
B_{3}=\left[\begin{array}{ccccc}
2 n-8 & 1 & 1 & 0 & 0 \\
n-4 & n-2 & 1 & 0 & 1 \\
n-4 & 1 & n-2 & 1 & 0 \\
0 & 0 & 1 & 2 & 1 \\
0 & 1 & 0 & 1 & 2
\end{array}\right] \begin{gathered}
S_{0} \\
S_{1} \\
v \\
v
\end{gathered}
$$

By simple computation, the characteristic polynomial of $B_{3}$ is $\phi\left(B_{3}, x\right)=f_{1}(x) f_{2}(x)$, where $f_{1}(x)=x^{3}-(3 n-6) x^{2}+\left(2 n^{2}-3 n-12\right) x-6 n^{2}+38 n-56$ and $f_{2}(x)=$ $x^{2}-(n-2) x+n-4$. Let $\gamma_{1} \geq \gamma_{2} \geq \gamma_{3}$ be the three roots of $f_{1}(x)$, and $\gamma_{1}^{\prime} \geq \gamma_{2}^{\prime}$ the two roots of $f_{2}(x)$. We have $\gamma_{1}>2 n-6$ because $f_{1}(2 n-6)=-4 n+16<0$, and $\gamma_{1}^{\prime}=\frac{n-2+\sqrt{n^{2}-8 n+20}}{2}, \gamma_{2}^{\prime}=\frac{n-2-\sqrt{n^{2}-8 n+20}}{2}$. Also, since $f_{1}\left(\gamma_{1}^{\prime}\right)=-2(n-4)(n-3-$ $\left.\sqrt{n^{2}-8 n+20}\right)<0$, we obtain $\gamma_{1}>\gamma_{1}^{\prime}>\gamma_{2}$ or $\gamma_{1}^{\prime}<\gamma_{3}$. We claim that the later case cannot occurs, since otherwise we have $4 n-8=\operatorname{trace}\left(B_{3}\right)>\gamma_{1}+3 \gamma_{1}^{\prime}+\gamma_{2}^{\prime}>4 n-10+$ $\sqrt{n^{2}-8 n+20}$, a contradiction. Therefore, we have $q_{2}(\bar{G})=\gamma_{1}^{\prime}=\frac{n-2+\sqrt{n^{2}-8 n+20}}{2}$, and so $q_{2}(G)+q_{2}(\bar{G})=n-2+\sqrt{n^{2}-8 n+20}<2 n-5$.

Case 2. $s_{1}=0$ and $s_{2} \geq 1$.

If $s_{2} \geq 2$, then $G$ is a spanning subgraph of $H(n-4,0,2)$. We see that $H(n-$ $4,0,2)$ has $Q$-eigenvalues 2 of multiplicity at least $n-5,1$ of multiplicity at least 
one, and the remaining four $Q$-eigenvalues, denoted by $\alpha_{1}>\alpha_{2} \geq \alpha_{3}>\alpha_{4}=0$, are in the quotient matrix

$$
B_{1}=\left[\begin{array}{cccc}
2 & 0 & 1 & 1 \\
0 & 1 & 0 & 1 \\
n-4 & 0 & n-4 & 0 \\
n-4 & 2 & 0 & n-2
\end{array}\right] \begin{gathered}
S_{0} \\
S_{2} \\
u \\
v
\end{gathered}
$$

By simple computation, the characteristic polynomial of $B_{1}$ is $\phi\left(B_{1}, x\right)=x f(x)$, where $f(x)=x^{3}-(2 n-3) x^{2}+\left(n^{2}-2 n-2\right) x-n^{2}+4 n$. Similarly, as $f(n-3)=$ $-n+6 \leq 0$, we have $\alpha_{2} \leq n-3$ or $\alpha_{3} \geq n-3$. We claim that the later case cannot occurs, since otherwise we have $2 n-3=\operatorname{trace}\left(B_{1}\right)=\alpha_{1}+\alpha_{2}+\alpha_{3}+\alpha_{4}>$ $3 \alpha_{3} \geq 3(n-3)$, a contradiction. Thus $q_{2}(G) \leq q_{2}(H(n-4,0,2))=\alpha_{2} \leq n-3$. Furthermore, by Lemma 2.8, we have $q_{2}(\bar{G})<n-2$ because $G$ is connected but not balanced. Thus $q_{2}(G)+q_{2}(\bar{G})<2 n-5$. If $s_{2}=1$, then $G=H(n-3,0,1)$. For $n=6,7$, one can easily check that $q_{2}(G)+q_{2}(\bar{G})<2 n-5$. Now suppose $n \geq 8$. We see that $G$ has $Q$-eigenvalue 2 of multiplicity at least $n-4$, and the remaining four $Q$-eigenvalues, denoted by $\beta_{1}>\beta_{2} \geq \beta_{3}>\beta_{4}=0$, are contained in the quotient matrix

$$
B_{2}=\left[\begin{array}{cccc}
2 & 0 & 1 & 1 \\
0 & 1 & 0 & 1 \\
n-3 & 0 & n-3 & 0 \\
n-3 & 1 & 0 & n-2
\end{array}\right] \begin{gathered}
S_{0} \\
S_{2} \\
u \\
v
\end{gathered}
$$

The characteristic polynomial of $B_{2}$ is $\phi\left(B_{2}, x\right)=x g(x)$, where $g(x)=x^{3}-(2 n-$ 2) $x^{2}+\left(n^{2}-n-2\right) x-n^{2}+3 n$. Since $g\left(n-\frac{5}{2}\right)=\frac{-2 n+15}{8}<0$, as above, we have $q_{2}(G)=\beta_{2}<n-\frac{5}{2}$. Now consider the complement graph $\bar{G}=\overline{H(n-3,0,1)}$. We see that $\bar{G}$ has $Q$-eigenvalue $n-4$ of multiplicity at least $n-4$, and the remaining four $Q$-eigenvalues, denoted by $\gamma_{1}>\gamma_{2} \geq \gamma_{3} \geq \gamma_{4}>0$ lie in the quotient matrix

$$
B_{3}=\left[\begin{array}{cccc|c}
2 n-7 & 1 & 0 & 0 \\
n-3 & n-2 & 1 & 0 & S_{0} \\
S_{2} \\
0 & 1 & 2 & 1 \\
u \\
v
\end{array}\right.
$$

The characteristic polynomial of $B_{3}$ is $\phi\left(B_{3}, x\right)=x^{4}-(3 n-6) x^{3}+\left(2 n^{2}-3 n-10\right) x^{2}-$ $\left(6 n^{2}-35 n+48\right) x+2 n^{2}-14 n+24$. Also, we have $\phi\left(B_{3}, 2 n-6\right)=-4(n-3)(n-4)<0$, and $\phi\left(B_{3}, n-\frac{5}{2}\right)=-\frac{1}{16}\left((2 n-11)\left(4 n^{2}-24 n+39\right)\right)<0$, which implies that $\gamma_{1}>$ $2 n-6$ and $\gamma_{2}<n-\frac{5}{2}$. Thus $q_{2}(\bar{G})=\max \left\{n-4, \gamma_{2}\right\}<n-\frac{5}{2}$. Hence, we get $q_{2}(G)+q_{2}(\bar{G})<2 n-5$.

Concluding the above results, we obtain that $q_{2}(G)+q_{2}(\bar{G}) \leq 2 n-5$, where the equality holds if and only if $G$ is one of the graphs shown in Figure 2.

We complete the proof.

Proof of Theorem 1.5. Clearly, we have $q_{2}(G)+q_{2}(\bar{G}) \leq 2 n-5$, where the equality holds if and only if $q_{2}(G)=n-3$ and $q_{2}(\bar{G})=n-2$. If $q_{2}(\bar{G})=n-2$, by Lemma 2.8, the connected graph $G$ must be bipartite and balanced. Thus the results follows from the proof of Theorem 1.4 immediately. 
Acknowledgements. The first author is supported by the National Natural Science Foundation of China (No. 11671344), the China Postdoctoral Science Foundation and the Postdoctoral Research Sponsorship in Henan Province (No. 1902011). The second author is supported by the National Natural Science Foundation of China (Nos. 11771141) and Fundamental Research Fund for the Central Universities (No. 222201714049).

\section{References}

[1] N. Abreu, A.E. Brondani, L.S. de Lima, C. Oliveira, A note on the NordhausGaddum type inequality to the second largest eigenvalue of a graph, Appl. Anal. Discrete Math. 11 (2017) 123-135.

[2] M. Aouchiche, P. Hansen, A survey of Nordhaus-Gaddum type relations, Discrete Appl. Math. 161 (2013) 466-546.

[3] F. Ashraf, B. Tayfeh-Rezaie, Nordhaus-Gaddum type inequalities for Laplacian and signless Laplacian eigenvalues, Electron. J. Combin. 21 (3) (2014) \#P3.6.

[4] A.E. Brouwer, W.H. Haemers, Spectra of Graphs, Springer, Berlin, 2011.

[5] P. Csikvári, On a conjecture of V. Nikiforov, Discrete Math. 309 (2009) 4522 4526 .

[6] X. Chen, K.C. Das, Some results on the Laplacian spread of a graph, Linear Algebra Appl. 505 (2016) 245-260.

[7] K.C. Das, On conjectures involving second Largest signless Laplacian eigenvalue of graphs, Linear Algebra Appl. 432 (2010) 3018-3029.

[8] M. Einollahzadeh, M.M. Karkhaneei, Proof of a conjecture on the algebraic connectivity of a graph and its complement, arXiv:1901.02047 [math.CO].

[9] L.S. de Lima, V. Nikiforov, On the second largest eigenvalue of the signless Laplacian, Linear Algebra Appl. 438 (2013) 1215-1222.

[10] L. Feng, G. Yu, On three conjectures involving the signless Laplacian spectral radius of graphs, Publ. Inst. Math. (Beograd) (N.S.) 85 (99) (2009) 35-38.

[11] S. Guo, Y. Chen, G. Yu, A lower bound on the least signless Laplacian eigenvalue of a graph, Linear Algebra Appl. 448 (2014) 217-221.

[12] C.D. Godsil, G. Royle, Algebraic Graph Theory, in: Graduate Texts in Mathematics, vol. 207, Springer, New York, 2001.

[13] W.H. Haemers, Interlacing eigenvalues and graphs, Linear Algebra Appl. 226228 (1995) 593-616.

[14] J.V.D. Heuvel, Hamilton cycles and eigenvalues of graphs, Linear Algebra Appl. 226-228 (1995) 723-730.

[15] V. Nikiforov, Eigenvalue problems of Nordhaus-Gaddum type, Discrete Math. 307 (6) (2007) 774-780.

[16] V. Nikiforov, X. Yuan, More eigenvalue problems of Nordhaus-Gaddum type, Linear Algebra Appl. 451 (2014) 231-245. 
[17] E. Nordhaus, J. Gaddum, On complementary graphs, Amer. Math. Monthly 63 (1956) 175-177.

[18] E. Nosal, Eigenvalues of graphs, Master Thesis, University of Calgary, 1970.

[19] W. So, Commutativity and spectra of Hermitian matrices, Linear Algebra Appl. 212-213 (1994) 121-129.

[20] W.A. Stein et al., SageMath, The Sage Mathematics Software System (Version 8.7), 2019. http://www. sagemath.org.

[21] T. Terpai, Proof of a conjecture of V. Nikiforov, Combinatorica 31 (2011) 739 754.

[22] Z. You, B. Liu, The Laplacian spread of graphs, Czechoslovak Math. J. 62 (137) (2012) 155-168.

[23] M. Zhai, J. Shu, Y. Hong, On the Laplacian spread of graphs, Appl. Math. Lett. 24 (2011) 2097-2101. 